\title{
Tests of the Rescorla-Wagner model of Pavlovian conditioning
}

\author{
LEE LEVITAN \\ University of Minnesota, Minneapolis, Minnesota 55455
}

\begin{abstract}
The present experiment tested several predictions from the Rescorla-Wagner (1972) model of Pavlovian conditioning. For two groups, each of two CSs was independently paired with shock until asymptotic suppression developed. They were then presented simultaneously for a second phase: for one group, US intensity was not changed between phases, while for the second group it was increased. The compound lost associative strength in the first group but not in the second. For a third group, the first phase was run using only one CS. During compound conditioning, the same US was used to reinforce the compound formed by adding a novel CS; no change in suppression was seen during compound conditioning. Other predictions were made concerning additive summation, and these were confirmed, as were predictions concerning a test phase in which the CSs were again presented singly. The evidence is taken as strong support for the Rescorla-Wagner model.
\end{abstract}

According to a recent model of Pavlovian conditioning proposed by Rescorla and Wagner (1972), the change in associative strength $(\Delta \mathrm{V})$ accruing to a conditioned stimulus (CS) as the result of pairing with the unconditioned stimulus or US (i.e., a reinforced trial), is determined by the discrepancy between the asymptotic associative strength the US will support and the current associative strength of the stimulus complex in which the CS is embedded $\left(V_{\text {total }}\right)$. The discrepancy between the asymptote and $V_{\text {total }}$ determines the direction and magnitude of $\Delta \mathrm{V}:$ (a) if this discrepancy is positive, CS will increase in associative strength on that trial; (b) if this discrepancy is negative, CS will decrease in associative strength; and (c) if this discrepancy is zero, no change in associative strength will result.

Given these basic rules, it is possible to imagine situations in which reinforced trials can lead to decrements in the associative strength of CS. For example, suppose two CSs, A and B, are independently paired with shock (US) until the associative strength of each CS ( $V_{A}$ and $V_{B}$, respectively) is near asymptote. If we then present the two CSs in a simultaneous compound and continue to reinforce the compound with the same US used for single stimulus conditioning, what does the Rescorla-Wagner (1972) model predict will happen?

To answer this question, we first need some rule for determining the associative strength of a compound, given the strengths of its elements. The simplest assumption is that the associative strengths add

This research was supported by Grant PHS-5R01-MH18053 to Milton A. Trapold, who sponsors this research and takes full editorial responsibility for its content. The author gratefully acknowledges the assistance of Professors Trapold and J. Bruce Overmier for reviewing earlier drafts of this article. The author is now at: Division of Social Sciences, University of Minnesota, Morris, Morris, Minnesota 56267. algebraically, so that $V_{A}+V_{B}=V_{A B}$; this is the assumption made by Rescorla and Wagner (1972). Given this, two predictions then follow: (a) the total associative strength of the $\mathrm{AB}$ compound should exceed that of the individual elements, and $A B$ should initially have greater associative strength than either element alone; and (b) if we continue to reinforce $A B$ with the same US, one or both elements should lose associative strength. This second follows because, should $V_{A B}$ exceed the asymptote which the US will support, the discrepancy between asymptotic associative strength and $\mathrm{V}_{\mathrm{AB}}$ would be negative, and further reinforcements should lead to decrements in the associative strength of the compound. The model does not demand that both CSs lose associative strength to the same degree, because the magnitude of $\Delta \mathrm{V}$ on any trial is also assumed to be a function of stimulus salience. Thus, depending on the relative saliences of $A$ and $B$, one or both should come to control less suppression if this procedure is carried out long enough. This prediction has, in fact, been confirmed by Rescorla (1970, Experiment 2).

The present study was intended to replicate and extend Rescorla's findings. The extension is based upon the following analysis derived from the model: If, following independent conditioning of $\mathrm{A}$ and $\mathrm{B}$, we then present the $\mathrm{AB}$ compound, and we also increase US intensity during compound conditioning such that the new asymptote approximates $V_{A B}$, neither element should lose associative strength-the discrepancy between asymptote and $V_{A B}$ should be zero. The logical problem with attempting such a manipulation is that we do not know what the scaling function is for shock intensity and perceived magnitude, and consequently it is not clear what US intensity would be appropriate for compound conditioning. However, we could test the model by increasing US intensity for compound 
conditioning by some (objectively) significant amount. In such cases, the prediction is that the $\mathrm{AB}$ compound should not lose as much associative strength as in the group for which US intensity was not changed (i.e., in the group intended to replicate Rescorla's [1970, Experiment 2] results). Compound conditioning with an increased US intensity constituted a second group in the present study. Finally, in such a study, one must control for the effects of compounding per se. Thus, for a third group, only one of the CSs was used during single stimulus conditioning; in the second phase, the $\mathrm{AB}$ compound (where $\mathrm{A}$ was a novel $\mathrm{CS}$ ) was reinforced with the same US as used with B in the previous phase. This procedure also provides a third test of the Rescorla-Wagner (1972) model. That is, during compound conditioning, the associative strength of neither A nor B should change, because $V_{A B}$ should be near asymptote due to the $\mathrm{B}$ alone + shock training in the preceding phase.

\section{METHOD}

\section{Subjects}

The subjects were 24 experimentally naive male albino rats, purchased from Thorp Industries (Minneapolis, Minnesota). All subjects weighed $250-300 \mathrm{~g}$ upon arrival in the lab and were maintained at $80 \%$ of their ad-lib body weights throughout the experiment by controlled daily feedings. Water was continuously available in the home cages.

During the course of the experiment, two subjects died and five others failed to meet the Phase 1 conditioning criterion. Data from the remaining 17 subjects were analyzed.

\section{Apparatus}

The apparatus consisted of two identical operant conditioning chambers, $38 \times 38 \times 38 \mathrm{~cm}$, each enclosed in its own sound-attenuating structure. On the front wall of each chamber was a single retractable response lever, $5 \mathrm{~cm}$ wide, located $8 \mathrm{~cm}$ above the floor. The foodcup was located $11 \mathrm{~cm}$ to the right of the lever; all reinforcement consisted of the delivery of a single 45-mg Noyes food pellet. Each chamber was also equipped with a houselight, located directly above the foodcup, and a speaker in the ceiling.

Standard electromechanical programming and recording equipment was located in an adjacent room.

\section{Procedure}

Except for a delay between the end of single stimulus conditioning and the beginning of compound stimulus conditioning for some subjects (see below), subjects were run at approximately the same time each day on consecutive days, except in cases of mechanical failure or other unusual circumstances. Each subject was assigned to one of the two chambers at the outset of the experiment, and all sessions were run in that chamber.

Preliminary training. All subjects were first given two 30-min habituation sessions during which they were placed in the chamber with the houselight on and the lever retracted. Next, they were given two 60-min sessions of magazine training during each of which food pellets were delivered according to a variable-time 60 -sec schedule.

On the 5th day, the lever was in the chamber for the first time. Subjects were given one session of leverpress training on continuous reinforcement, to a criterion of 50 responses.
Finally, responding on a variable-interval (VI) 120-sec schedule was shaped in successive stages. Each subject was given a single 60-min session each on VI 30-sec and VI 60-sec, two sessions on VI 90-sec, and 14 sessions on VI 120-sec.

All subsequent phases consisted of 60 -min sessions.

Single stimulus conditioning. In this phase, aversive Pavlovian conditioning was carried out while the subject was leverpressing for food on the VI 120 -sec schedule. In such a conditioned suppression paradigm (see Estes \& Skinner, 1941), a neutral stimulus (the CS) is paired in time with an aversive US (here, shock), and it comes to evoke a reduction in instrumental response rate by itself. The degree of suppression is taken as an index of the degree of association (V) between the CS and US. The typical measure of degree of suppression produced by the CS is a suppression ratio (SR); the SR used in the present experiment was $S R=D /(P+D)$, where $D$ is instrumental response rate during the $C S$ and $P$ is rate in a comparable period of time just preceding CS onset. The nature of this SR is such that a value of .50 indicates the CS had no effect on response rate, while values less than .50 indicate suppression (Annau \& Kamin, 1961).

Subjects were divided into three groups: For both Group 1 $(N=6)$ and Group $2(N=5)$, two of four trials each day consisted of a tone followed by shock, while the other two trials consisted of the houselight flashing at $2 \mathrm{~Hz}$ followed by shock. For Group $3(\mathrm{~N}=6)$, two tone-shock trials were presented per session; the light CS was not presented for this group. Each CS presentation lasted $120 \mathrm{sec}$, with offset of the CS and onset of the .5 -sec shock concurrent.

Criterion for this phase was 3 consecutive days over which the average SR for each CS was between .10 and .40 . Shock intensity was adjusted for each subject individually to accomplish this.

Compound stimulus conditioning. This phase began when all subjects had completed the previous phase, which meant some subjects experienced time delays over which no training was given. The lag between phases was 2-25 days (mean: 15.4; standard deviation: 6.6); there is evidence that CSs retain their capacity to suppress instrumental responding over such delays-even for as long as 2.5 years (Hoffman, Fleshler, \& Jensen, 1963)-with no apparent loss in strength.

For all subjects, the tone and light now were presented simultaneously, followed by shock. In all cases, shock intensity was the same as that at which criterion for the previous phase was reached. For Groups 1 and 3, a single .5-sec shock was presented immediately at offset of the 120 -sec compound CS. For Group 2, a first shock was followed $.5 \mathrm{sec}$ after its termination by a second of the same intensity and duration. Compound conditioning continued for 14 sessions.

Testing. For all subjects, testing consisted of presentation of two tone CS trials and two light CS trials, each $120 \mathrm{sec}$ in duration, not followed by shock. Testing was carried out in a single session.

The plan of the experiment is summarized in Table 1.

\section{RESULTS AND DISCUSSION}

There was considerable variability in number of days for subjects to reach criterion during single stimulus conditioning. Overall, the range was $9-44$ days (mean: 22.9; standard deviation: 11.4). In addition, the range of shock intensities necessary to produce criterion performance was also surprisingly large: $.3-2.5 \mathrm{~mA}$ (mean: $.97 \mathrm{~mA}$ ). Also, it was pointed out earlier that no subject was begun on compound conditioning until all had reached criterion for single stimulus conditioning. 


\section{Single Stimulus Conditioning}

On the last day of this phase, Groups 1 and 2 did not differ in suppression to the light (CS A), $F(1,9)=3.33$. Further, there were no apparent differences among the three groups in suppression to the tone (CS B), F $(2,14)$ $=1.00$.

\section{Compound stimulus conditioning}

Subjects in both Groups 1 and 2 received conditioned suppression training to CS A and to CS B during single stimulus conditioning. If the associative strengths of $A$ and $B$ sum algebraically to produce the associative strength of the $\mathrm{AB}$ compound, as Rescorla and Wagner (1972) suggest, we would expect $A B$ to initially produce more suppression than was produced by $\mathrm{A}$ or B alone (see Weiss, 1972). To test this prediction, the lowest SR (indicating the greatest suppression) to either A or B was taken for each subject in these groups for each of the last 2 days of single stimulus conditioning and the mean compared to the mean $\mathrm{SR}$ to $\mathrm{AB}$ over the first 2 days of compound conditioning (see Figure 1). Pairwise comparisons confirmed the Rescorla-Wagner prediction for both Groups 1 and 2, viz., the $\mathrm{AB}$ compound produced significantly more suppression than had been produced by the strongest single element, $t(14)=4.03$ and 2.86 , respectively; ps $<.02$.

For Group 1 during compound conditioning, $\mathrm{AB}$ was reinforced with the same US as used to reinforce $A$ and $B$ independently during the previous phase. Given stable suppression to both $\mathrm{A}$ and $\mathrm{B}$ at the end of Phase 1, and given that the $\mathrm{AB}$ compound initially produced more suppression than either element alone, we can assume that $\mathrm{V}_{\mathbf{A B}}$ initially exceeded the asymptote for that US. Consequently, the discrepancy between asymptote and $V_{A B}$ was initially negative, and $A B+$ shock trials should have resulted in decrements in the strength of the compound. Thus, the compound should have produced less suppression at the end of compound stimulus conditioning than at the beginning of that phase. To test this, the mean $S R$ to $A B$ over the first 2 days of compound conditioning was compared to the mean for the last 2 days. Subsequent comparison confirmed that there was significant loss of suppression to $\mathrm{AB}$ in Group 1, $\mathrm{t}(14)=4.45, \mathrm{p}<.001$ (see Figure 1). Thus,

Table 1

Summary of Conditioning and Testing

\begin{tabular}{|c|c|c|c|c|}
\hline \multirow[b]{2}{*}{ Group } & \multirow[b]{2}{*}{$\mathbf{N}$} & \multicolumn{2}{|c|}{ Conditioning } & \multirow[b]{2}{*}{ Testing } \\
\hline & & Single Stimulus & Compound & \\
\hline 1 & 6 & $T+s k, L+s k$ & $\mathrm{TL}+\mathrm{sk}$ & $\mathrm{T}$ alone, $\mathrm{L}$ alone \\
\hline 2 & 5 & $\mathrm{~T}+\mathrm{sk} ; \mathrm{L}+\mathrm{sk}$ & TL + sk-sk* & $\mathrm{T}$ alone, $\mathrm{L}$ alone \\
\hline 3 & 6 & $\mathrm{~T}+\mathbf{s k}$ & $\mathrm{TL}+\mathrm{sk}$ & $\mathrm{T}$ alone, $\mathrm{L}$ alone \\
\hline
\end{tabular}

Note-T = tone, $L=$ light, sk = shock .

*For this group, one shock was followed .5 sec later by a second of equal intensity and duration.

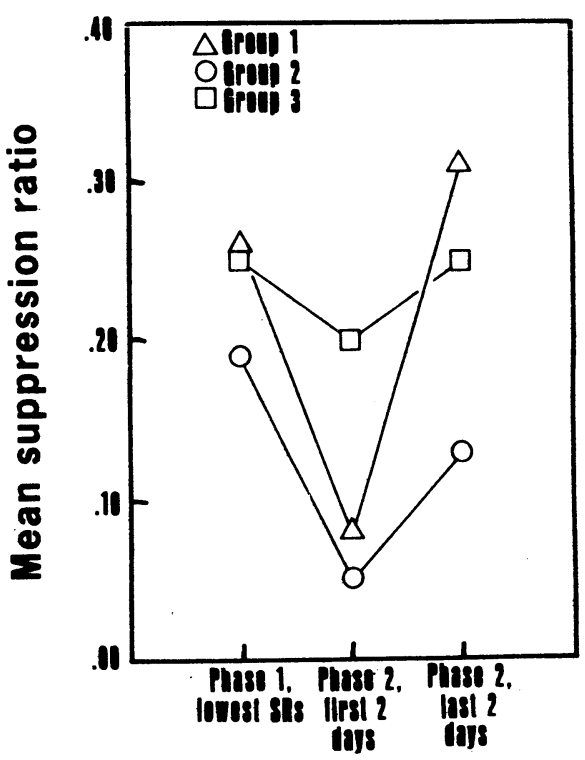

Figure 1. Mean greatest suppression to single CSs (Phase 1) and mean suppression to the compound CS (Phase 2).

under the conditions of this experiment, reinforced presentations of a CS resulted in decrements in the associative strength of that CS, as predicted by Rescorla and Wagner (1972).

In Group 2, the $\mathrm{AB}$ compound was reinforced with a more intense US than was used for independently conditioning $A$ and $B$, viz, two consecutive shocks separated by $.5 \mathrm{sec}$. Presumably, there should have been less recovery from suppression to $A B$ in this group than in Group 1. In fact, the two-shock procedure was quite successful: a $t$ test based on the ANOVA, comparing mean suppression to $\mathrm{AB}$ over the first 2 days and the last 2 days of compound conditioning (see Figure 1), confirmed the impression that there was no reliable loss of suppression to $A B$ in Group 2, $t(14)=1.41$. Again, this result provides strong support for the Rescorla-Wagner (1972) model.

Finally, for subjects in Group 3, only one CS was used during single stimulus conditioning. Introduction of the novel stimulus $(\mathrm{V}=0)$ during compounding should have had no significant effect on the degree of suppression produced: that is, the compound should have produced the same degree of suppression initially as did the originally trained element alone. Comparison following the ANOVA indicated that, as predicted, there was no change in suppression as a result of compounding the excitatory B stimulus with the neutral A stimulus, $t(14)=1.12$. Further, because the same US was used to reinforce $A B$ as had been used previously to reinforce $B$ alone, the initial value of $V_{A B}$ should have approximated asymptotic strength for the US, and thus $\mathrm{AB}$ should have produced the same degree of suppression at the end of compound conditioning as it had at the beginning. Comparison of the mean SR to $A B$ 


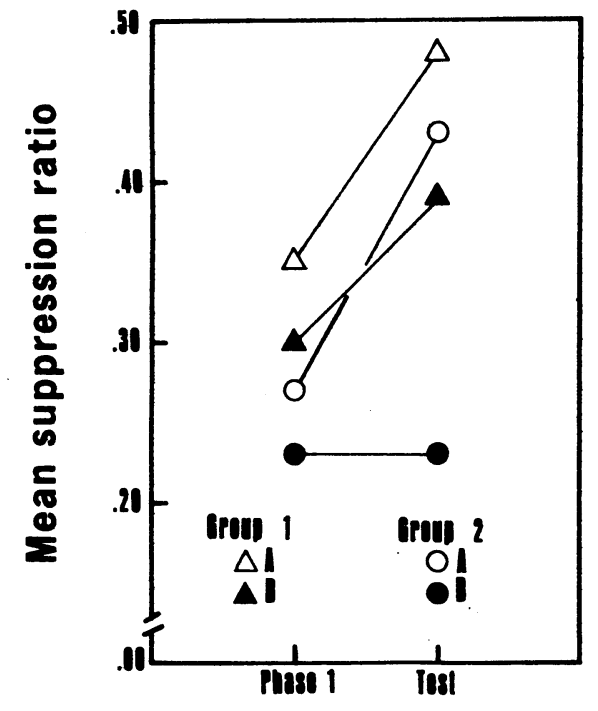

Figure 2. Mean suppression to each CS during single stimulus conditioning (Phase 1) and testing.

over the first 2 days and last 2 days of compound conditioning (see Figure 1) confirmed this prediction, $\mathrm{t}(14)=.97$.

\section{Testing of Elements}

During compound conditioning, there was loss of suppression produced by $\mathrm{AB}$ in Group 1 , but not in Group 2. Because the compound produced the same degree of suppression in both groups at the beginning of compound conditioning, the reduction of suppression to $\mathrm{AB}$ in Group 1 implies that, during testing, either CS A or CS B should have produced less suppression in Group 1 than in Group 2. Although we cannot say a priori which CS should have lost associative strength in Group 1, we can predict that the total of the suppression produced by CSs A and B individually should have been greater in Group 2 than in Group 1 during testing. To test this, a two groups by two phases (single stimulus conditioning vs. testing) by two stimuli ANOVA, with repeated measures on the last two factors, was run using data from the last day of single stimulus conditioning and from the test day. These data are shown in Figure 2.

Contrasts confirmed the impression that, at the end of single stimulus conditioning, total suppression to $A$ and $B$ was the same in both groups, $t(36)=1.58$, and supported the prediction that total suppression should be greater in Group 2 than in Group 1 during testing, $t(36)=2.17, p<.05$. In fact, inspection of Figure 2 shows that compound conditioning during Phase 2 had no effect on mean suppression to CS B in Group 2: the mean SRs at the end of single stimulus conditioning and at test were identical.

The present study replicates and extends Rescorla's (1970, Experiment 2) finding that reinforced presentations of a CS can, under the appropriate circumstances, result in decrements in the associative strength of that CS. All predictions were based on the model of Pavlovian conditioning proposed by Rescorla and Wagner (1972). Multiple predictions were made concerning subjects' behavior across three phases of the experiment, including predictions based on single stimulus presentations and compound stimulus presentations, as well as predictions concerning changes in behavior due to the nature of the reinforcing event. The fact that all predictions were confirmed is taken as strong evidence for the Rescorla-Wagner (1972) model.

\section{REFERENCES}

Annau, Z., \& Kamin, L. J. The conditioned emotional response as a function of intensity of the US. Journal of Comparative and Physiological Psychology, 1961, 54, 428-432.

Estes, W. K., \& Skinner, B. F. Some quantitative properties of anxiety. Journal of Experimental Psychology, 1941, 29. 390-400.

Hoffman, H. S., Fleshler, M., \& Jensen, P. Stimulus aspects of aversive controls: The retention of conditioned suppression. Journal of the Experimental Analysis of Behavior, 1963, 6, 575-583.

Rescorla, R. A. Reduction in the effectiveness of reinforcement after prior excitatory conditioning. Learning and Motivation, 1970, 1, 372-381.

Rescorla, R. A., \& WAgner, A. R. A theory of Pavlovian conditioning: Variations in the effectiveness of reinforcement and nonreinforcement. In A. H. Black \& W. F. Prokasy (Eds.), Classical conditioning II. New York: Appleton-Century-Crofts, 1972.

WEISS, S. J. Stimulus compounding in free-operant and classical conditioning. A review and analysis. Psychological Bulletin, 1972, 78, 189-208.

(Received for publication June 4, 1975.) 